\title{
Eclectic psychotherapy and case formulation
}

\author{
Cristian Vasile* \\ Petroleum Gas University of Ploiesti, Bd. Bucuresti, nr, 39, Ploiesti, 100680, Romania
}

\begin{abstract}
In the last two decades, the clinical efficacy of various psychotherapeutic schools has begun to be increasingly analyzed and discussed. Moreover, there have been additions to approaches that have already become classic or even new approaches in psychotherapy. In this context, integrative and/or systemic directions have been developed, which try to offer solutions on multiple levels at the individual and relational levels. Integrative psychotherapy attempts to put together various theoretical systems under a system that would be superordinate or metatheoretical. Synthetic psychotherapy tries to combine various approaches both technically and theoretically. Eclectic psychotherapy makes a selection of valid and useful methods and techniques from different approaches.

The eclectic approach in psychotherapy is part of this current, but also a necessity for streamlining interventions and working with clients.

Eclectic therapy in clinical practice is not a new direction, but we believe that especially therapists with rich experience in working with clients can successfully address it because it requires extensive and indepth knowledge of various methods and techniques from many approaches.
\end{abstract}

Keywords: eclectic psychotherapy; psychotherapy; efficacy; case formulation

\section{Introduction}

Until the 1970s, therapy schools were separate from each other, and therapists adhered to a specific approach. There were very few overlaps, and the professionals were loyal to the chosen method. Over time, research has continued, and as more therapeutic approaches have developed, people have begun to see that more types of therapy are effective, and since the 1970s, therapists have begun to use more approaches in their sessions with patients.

Arnold Lazarus, by introducing multimodal psychotherapy intervention (Lazarus, 1981), was one of the first specialists to propose an integrated approach to psychotherapy. Since the 1970s and 1980s, various types of eclectic therapy have been developed and continued to evolve. Today, new eclectic therapies continue to emerge as research continues and studies are carried out about which therapeutic elements determine that psychotherapy is effective for different persons, each person having unique problems.

\subsection{What is eclectic psychotherapy?}

Eclectic therapy is an approach that starts from other pre-existing theoretical and technical approaches. It is characterized by flexibility, uses several facets, which allows the specialist to intervene with the most effective methods to meet the needs of each client. It is also sometimes referred to as multimodal therapy or integrative psychotherapy, although these names are sometimes debatable.

\footnotetext{
* Corresponding author.

E-mail address: clinical_psycho@yahoo.com
} 
At the beginning of the twentieth century, with the advent of psychoanalysis, then influenced by other currents, many mental health specialists chose a single style of treatment (in that period sometimes there was rivalry between different schools of psychotherapy). In recent decades, however, more and more psychotherapists have begun to take over and use ideas from different therapeutic schools, by the needs they identified with the patient.

Studies indicate that most psychotherapists currently use an eclectic or integrative approach. Research conducted on this professional category indicates that only $15 \%$ of specialists used a single theoretical model and that an average of four therapeutic approaches was used in eclectic practice in a therapeutic approach (Tasca et al., 2015).

\section{Eclectic psychotherapy types}

With the focus on clinical efficacy and patient well-being, several types of eclectic therapy have emerged. The need for structuring and efficiency has led to the selection of some techniques or others from a wide range so that the therapeutic intervention can meet the unique needs of each patient. In each case, these approaches are based on a series of techniques to solve the unique needs of the individual. We will mention here: short eclectic therapy, cognitive-interpersonal therapy, multimodal therapy, trans-theoretical therapy.

Short eclectic therapy - is a form of short-term eclectic therapy, in which psychodynamic and cognitive-behavioral techniques are used in a structured way, in a limited number of sessions, often for a specific problem. This approach has been used with increased efficiency in PTSD, supporting patients in changing their view of the traumatic experience and in developing new coping strategies.

Cognitive-interpersonal therapy - starts from three aspects of cognitive-behavioral therapy, accepting the understanding of how patients' thoughts influence their social relationships.

In this approach, an essential role is assigned to interpersonal aspects and the therapeutic alliance to achieve the objectives of psychological intervention.

Safran and Segal's cognitive-interpersonal therapy (CIT) (Safran and Segal, 1990) emphasizes the role of the therapeutic relationship between therapist and patient, as well as the nature of the relationship between cognitive and interpersonal processes. The therapist does not have an objective and neutral role but is seen as a human being with difficulties and strengths, who will interact with the problems and weaknesses of the patient.

Three specific mechanisms of change are used in the cognitive-interpersonal therapy of Safran and Segal. These will lead to the development of a new experience for the patient:

1. Decentralization

The patient is directed to experience his role in the construction of his reality.

2. Experiential denial

It refers to the rejection of dysfunctional beliefs about oneself and others, through the view of new experiences.

3. Access to information on provisions for action

The mechanism involves the discovery of unaware aspects of the patient's experience, or his inner life.

The purpose of the three mechanisms is not so much the understanding or rational analysis of reality, but the identification of new ways to experience reality, about oneself and others.

Multimodal therapy - the approach starts from elements of social-cognitive learning and uses several techniques from other therapies. Lazarus (1981) considers that human beings function at the biological, sensory-perceptual, cognitive, emotional, and social levels, and each of these parts needs to be addressed in psychotherapeutic 
practice. We mention here the multimodal intervention model BASIC I.D. (behavior, affect, sensation, imagery, cognition, interpersonal relationship, drugs-biology).

Trans-theoretical therapy - aims to understand the stages of change, as well as the process of making a change. Based on this knowledge, patients become able to move effectively towards achieving their goals, improve their interpersonal relationships, and create positive changes in their lives.

Prochaska suggests that in a natural way humans use ten different changing processes to modify the negative behavior, while therapeutic systems use only two or three processes (Xiao et al., 2001). From this perspective, therapists should be able to use a similar number of processes, at the cognitive level, to be able to work effectively with their patients in the trans-theoretical approach.

A second important element in the trans-theoretical approach refers to the stage of change, which reflects motivational and time-related aspects. Intentional change takes place gradually, through several stages, and Prochaska has identified five basic stages: pre-contemplation, contemplation, preparation, action, and maintenance. A stage of change is characterized by both a period and the package of tasks required to move on to the next stage. The third element of the trans-theoretical approach analyzes the levels of change.

The model of the stages of change of Prochaska and DiClemente was later revised, and new stages were added, increasing the specificity of the model (Dolan et al., 2006).

The increased flexibility of eclectic psychotherapy does not mean that the specialist does not have to work on an individualized intervention plan for each patient, starting from the analysis of the unique needs of the patient, but also the specificity of each technique.

\subsection{Included approaches and addressed issues}

Theoretical approaches that could be identified in the eclectic orientation may include:

- $\quad$ Psychodynamic therapy

- Behavioral therapy

- $\quad$ Cognitive-behavioral therapy (CBT)

- $\quad$ Therapy based on mindfulness

- Interpersonal therapy

- $\quad$ Dialectical-behavioral therapy (DBT)

- $\quad$ Person-centered therapy

- Guided imagery

Problems that can be addressed through eclectic psychotherapy

The eclectic approach requires in-depth knowledge of the patient (an anamnesis analysis is required), detailed knowledge of techniques from various orientations, both theoretically and practically, and the creation of an individualized intervention plan that can address one or more of the following disorders, but not limited to them:

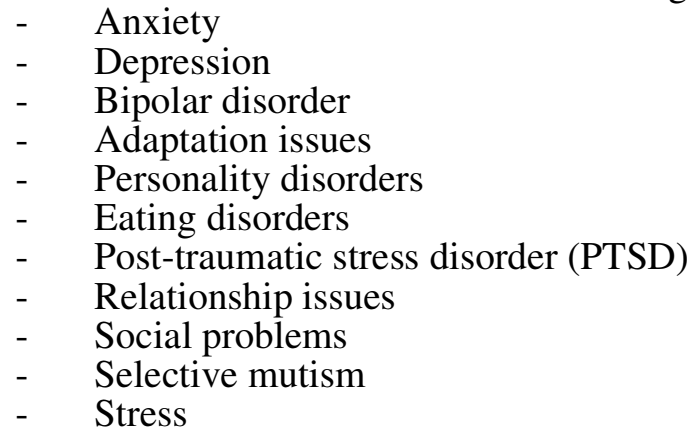




\section{ET advantages, disadvantages and efficacy studies}

Eclectic therapy can be analyzed in terms of important benefits such as:

- Individualized approach - each patient is unique, with its own life experience, its interpretations of various events, and its structure of personality. From this perspective, the methods and techniques used in eclectic approach are much more diverse and adaptable than if the techniques of a single therapeutic school were used. Thus, the intervention plan is individualized and can be adapted along the way, depending on the evolution of the therapeutic approach.

- Involvement - sometimes patients get bored when the same techniques are used constantly, repeatedly in therapy sessions. The use of various methods and techniques, in addition to increasing therapeutic efficacy, also has the advantage of increasing patient interest and involvement.

- Flexibility - the broad range of methods and techniques used by the therapist, makes it easier to change them or switch between them, in accordance with the evolution of the therapeutic process. Techniques considered more effective than others, or techniques that are better suited to the patient's personality and his problem are used. The techniques switch can occur to address one or more of the patient's problems.

\subsection{Efficacy}

Studies suggest that eclectic psychotherapy is a safe and highly effective form of intervention. An important factor that makes the difference between the results obtained by two or more therapists can be the experience of therapists, and this is an important variable in the psychotherapeutic process.

Eclectic psychotherapy is presented as an effective therapeutic approach in the case of selective mutism disorder, an anxiety disorder encountered in children who have a constant failure to speak in specific social situations. The intervention involved the use of psychodynamic, behavioral, cognitive-behavioral, and family techniques and strategies (Camposano, 2011).

According to another study (Gersons and Schnyder, 2013), techniques from psychodynamic and cognitive-behavioral approaches have been used effectively to build an eclectically successful intervention for PTSD.

Another study (Ahmadi et al., 2017) used art therapy and CBT with increased effectiveness of these techniques in improving mental health and reducing anxiety in children.

\subsection{Possible disadvantages of eclectic psychotherapy}

- $\quad$ Lack of structure. Although the intervention plan needs to be structured, if the specialist prefers a less structured approach, then the patient may find that the eclectic approach is less predictable and therefore more difficult to follow.

The method could be seen as working on the trial-error model. It may seem to the patient that the therapist uses several techniques to try to find what fits best the problem, and this can cause a dose of frustration to the patient. However, the possibility to change techniques is also an advantage, as we discussed above (flexibility).

The eclectic approach does not have a high degree of efficiency for all problems. For some disorders, a certain type of intervention works better (phobias, some personality disorders, and, specifically, those disorders or problems that are addressed with a high degree of effectiveness through a specific approach), it is more appropriate to use the specifically recommended mode of intervention as a primary approach.

- Coverage of health insurance costs. The use of techniques that have been taken from various psychotherapeutic schools, may affect the payment of expenses from the health insurance budget. In most countries, certain therapeutic approaches 
have been considered effective, following studies, and insurance reimburses expenses only for those approaches (e.g. CBT, as an evidence-based therapeutic approach).

Training of specialists. An eclectic psychotherapist should be trained in more therapeutic schools. This training, together with the therapist's experience, leads to increased efficiency in the therapeutic act. The training and experience of the therapist are fundamental variables that act in the direction of streamlining the intervention (the second variable refers to the techniques used concerning the patient's personality and his problem).

\section{Case formulation}

The case formulation has to take into account the therapeutic approach in which the specialist works. It is one of the most important stages of the therapeutic act. If it is not performed correctly, then the effectiveness of the intervention can decrease dramatically and the patient does not feel any improvement (or no significant improvement).

In the case of eclectic psychotherapeutic intervention, the case conceptualization should be approached with even greater attention, because several guidelines are used, i.e. those guidelines from which the specialist will choose techniques to assist the client. The case formulation or conceptualization is all the more important, as it involves the elaboration of an explanatory scheme about the patient's problems. Thus, a scheme or a map of the therapeutic intervention is made, and this could help a lot with the therapeutic approach. (Needleman, 2003).

George Kelly (founder of the theory of personality construction - the theory of personal constructs), believes that the clinical traditional diagnosis, by forced integration of each case in a clinical structure (clinical nosology) is not appropriate for psychotherapy because it does not streamline the therapeutic act. In his book The Psychology of Personal Constructs (1955), Kelly states that "Much of the reform proposed by the psychology of personal constructs is directed towards the tendency for psychologists to impose preemptive constructions upon human behavior. Diagnosis is all too frequently an attempt to cram a whole live struggling client into a nosological category". Instead of nosological categories, Kelly used the concept of 'formulation', showing that there are two stages in the formulation of the case:

the first stage of structuring, when the specialist organizes the clinical case information "in terms of dimensions rather than in terms of disease entities", analyzing "the more important ways in which the client can change, and not merely ways in which the psychologist can distinguish him from other persons" (Kelly, 1955);

the second stage of construction, the specialist follows the integration between the information about the case and the personal meanings of the client regarding his problem.

For an accurate understanding of the patient's problem, it is necessary to break it down into operationalized components, which can be measured and approached specifically. The multilevel analysis of a problem offers the first level of operationalization, by breaking it down into levels of manifestation: behavioral, cognitive, emotional, and physiological.

Hans Eysenck, Monte B. Shapiro, Vic Meyer have developed individualized systematic alternatives to psychodiagnostic. Moreover, Meyer used a case form model in a course he taught at Middlesex Hospital Medical School in London in 1970. He used the term "behavioral formulation" or "problem formulation" for clinical formulation.

Characteristics of the case conceptualization:

- $\quad$ summarizing the major problems of the patient;

- identifying relations between different problems of the patient, using theories from clinical and therapeutic approaches;

explaining, based on psychological theories, the origin, development, and maintenance of the patient's problems, by the present situation; 
- building an intervention plan based on the psychological processes and principles already identified;

- the formulation of the case remains open to revisions and reformulations, if or when the therapeutic situation requires it.

\subsection{The five $P$ of case formulation}

The case conceptualization/formulation will follow the five steps shown below (see also Fig. 1)

- Problem presentation: What issues does the patient discuss?

- Precipitating factors: What triggers/stimuli led the patient to the current state?

- $\quad$ Predisposing factors: family history, genetic factors, cultural beliefs, etc.

- Perpetuating factors: most often the social environment. What kept the patient in the discomfort zone?

- Protective factors: what strengths does the patient have or what resources could he access to manage the situation?

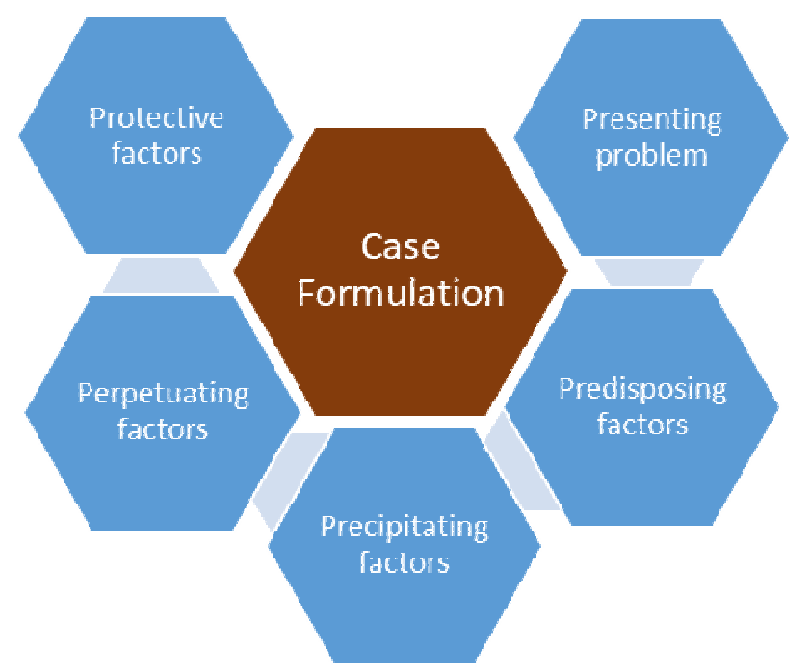

Fig. 1. Case formulation process

\section{Discussion}

The psychotherapy intervention through an eclectic approach seems to be the next level in psychotherapy, along with the integrative vision. It could be a very effective intervention if some important conditions are fulfilled:

- The psychotherapist has enough experience to draw and use the most appropriate techniques from different schools.

- The case formulation follows the information from the patient and the indirect data from his history. The conceptualization is structured and correct according to the planned methods and techniques from different approaches.

- Every patient is seen as a unique individual and the techniques used are chosen in accordance with the patient's personality and the problem specificity.

- $\quad$ Both the therapist and the patient are available to change the technique if no positive (or too little) change occurs. 


\section{References}

1. Ahmadi A, Mustaffa MS, Haghdoost AA, Mansor SMS. (2017). Eclectic approach to anxiety disorders among rural children. Trends Psychiatry Psychotherapy. 39(2):88-97. doi:10.1590/2237-6089-2016-0047.

2. British Psychological Society (2011). Good Practice Guidelines on the use of psychological formulation. Retrieved at http://www.bps.org.uk/system/files/Public files/DCP/cat-842.pdf.

3. Camposano L. (2011). Silent Suffering: Children with Selective Mutism. The Professional Counselor Volume 1, Issue 1 I Pages 46-56 doi:10.15241/lc.1.1.46.

4. Dolan, M.J., Seay, T.A., \& Vellela, T.C. (2006). The revised stage of change model and the treatment planning process. In G.R. Walz, J.C. Bleuer, \& R.K. Yep (Eds.). Vistas: Compelling perspectives on counseling 2006. Alexanderia, VA: American Counseling Association.

5. Gersons BP, Schnyder U. (2013). Learning from traumatic experiences with brief eclectic psychotherapy for PTSD. Eur J Psychotraumatol. 20:4. doi:10.3402/ejpt.v4i0.21369.

6. Kelly, George (1991) [1955]. The psychology of personal constructs: volume two: clinical diagnosis and psychotherapy. London; New York: Routledge in association with the Centre for Personal Construct Psychology. ISBN 9780415037983.

7. Lazarus, A. (1981). The Practice of Multimodal Therapy. New York, NY: McGraw-Hill.

8. Needleman, L. (2003). Case conceptualization in predicting and responding to therapeutic difficulties. In R. Leahy (Ed.), Overcoming resistance in cognitive therapy. New York: Guilford Press.

9. Safran, J.D., \& Segal, Z.V. (1990).Interpersonal process in cognitive therapy. New York: Basic Books

10. Tasca GA, Sylvestre J, Balfour L, et al. (2015). What clinicians want: Findings from a psychotherapy practice research network survey. Psychotherapy (Chic). 52(1):1-11. doi:10.1037/a0038252.

11.Xiao, J, O Neill, B, Prochaska, J, Kerbel, C, Brennan, P, Bristow, B. (2001). Application of The Transteorethical Model of Change To Financial Behavior. Consumer Interests Annual. 47. 1-9. 